Article

\title{
Covid-19 Transmission Trajectories-Monitoring the Pandemic in the Worldwide Context
}

\author{
Henry Loeffler-Wirth *, Maria Schmidt and Hans Binder *(D) \\ IZBI, Interdisciplinary Centre for Bioinformatics, Universität Leipzig, Härtelstr. 16-18, 04107 Leipzig, Germany; \\ schmidt@izbi.uni-leipzig.de \\ * Correspondence: wirth@izbi.uni-leipzig.de (H.L.-W.); binder@izbi.uni-leipzig.de (H.B.)
}

Received: 30 June 2020; Accepted: 14 July 2020; Published: 20 July 2020

\begin{abstract}
The Covid-19 pandemic is developing worldwide with common dynamics but also with marked differences between regions and countries. These are not completely understood, but presumably, provide a clue to find ways to mitigate epidemics until strategies leading to its eradication become available. We describe an iteractive monitoring tool available in the internet. It enables inspection of the dynamic state of the epidemic in 187 countries using trajectories that visualize the transmission and removal rates of the epidemic and in this way bridge epi-curve tracking with modelling approaches. Examples were provided which characterize state of epidemic in different regions of the world in terms of fast and slow growing and decaying regimes and estimate associated rate factors. The basic spread of the disease is associated with transmission between two individuals every two-three days on the average. Non-pharmaceutical interventions decrease this value to up to ten days, whereas 'complete lock down' measures are required to stop the epidemic. Comparison of trajectories revealed marked differences between the countries regarding efficiency of measures taken against the epidemic. Trajectories also reveal marked country-specific recovery and death rate dynamics. The results presented refer to the pandemic state in May to July 2020 and can serve as 'working instruction' for timely monitoring using the interactive monitoring tool as a sort of 'seismometer' for the evaluation of the state of epidemic, e.g., the possible effect of measures taken in both, lock-down and lock-up directions. Comparison of trajectories between countries and regions will support developing hypotheses and models to better understand regional differences of dynamics of Covid-19.
\end{abstract}

Keywords: pandemic dynamics; transmission rates; death toll; non-pharmaceutical interventions; epidemic mitigation; regional differences

\section{Introduction}

The coronavirus disease COVID-19 had spread to 187 countries with 5.5 Mio reported infections and more than 300,000 reported deaths worldwide by the end of May (25 May 2020) and 10.4 Mio reported infections and 510,000 reported deaths one month later (30 June 2020). The disease affects almost all spheres of life, especially public health, economics and well-being. The present situation and the near future (from months to one-two years) will require our coexistence with the virus until effective pharmaceutical countermeasures (medication, vaccine) are available and applicable [1,2]. This coexistence requires adjustment of a balance between a controllable low level of infections and maximum-possible levels of public life and economic activity.

Controlling the infection requires feedback loops sensitive to early and robust indications of secondary outbreak waves. This includes permanent surveillance of epidemiologic and medical indicators by testing programs, monitoring of case numbers and symptoms and forecasting methods on the one hand, and suitable 'no-pharmacological intervention' (NPIs) strategies on the other hand. 
Various 'number-tracker' tools are active (e.g., [3-7]). They mostly plot case numbers (infected, recovered, deaths) over time, usually on a country-by-country (or region-by-region) basis. As an illustration, we show the number of current infections and of Covid-19 related deaths as a function of time in selected countries (Figure 1). These 'epi-curves' reveal how the epidemic was expanding in time and space from China (at the end of 2019 and January 2020) via other Asian countries (South Korea, Iran) and Western Europe towards Eastern Europe, the Americas and other parts of the world (February to April 2020). They also reveal different phases of epidemic, namely, an initial 'take-off stage', an 'exponential growing stage' followed by 'slowed growth', 'turning into a decline' and 'decline' [5]. Charting the outbreak day by day in each country illustrates the global story of this succession of events [3].
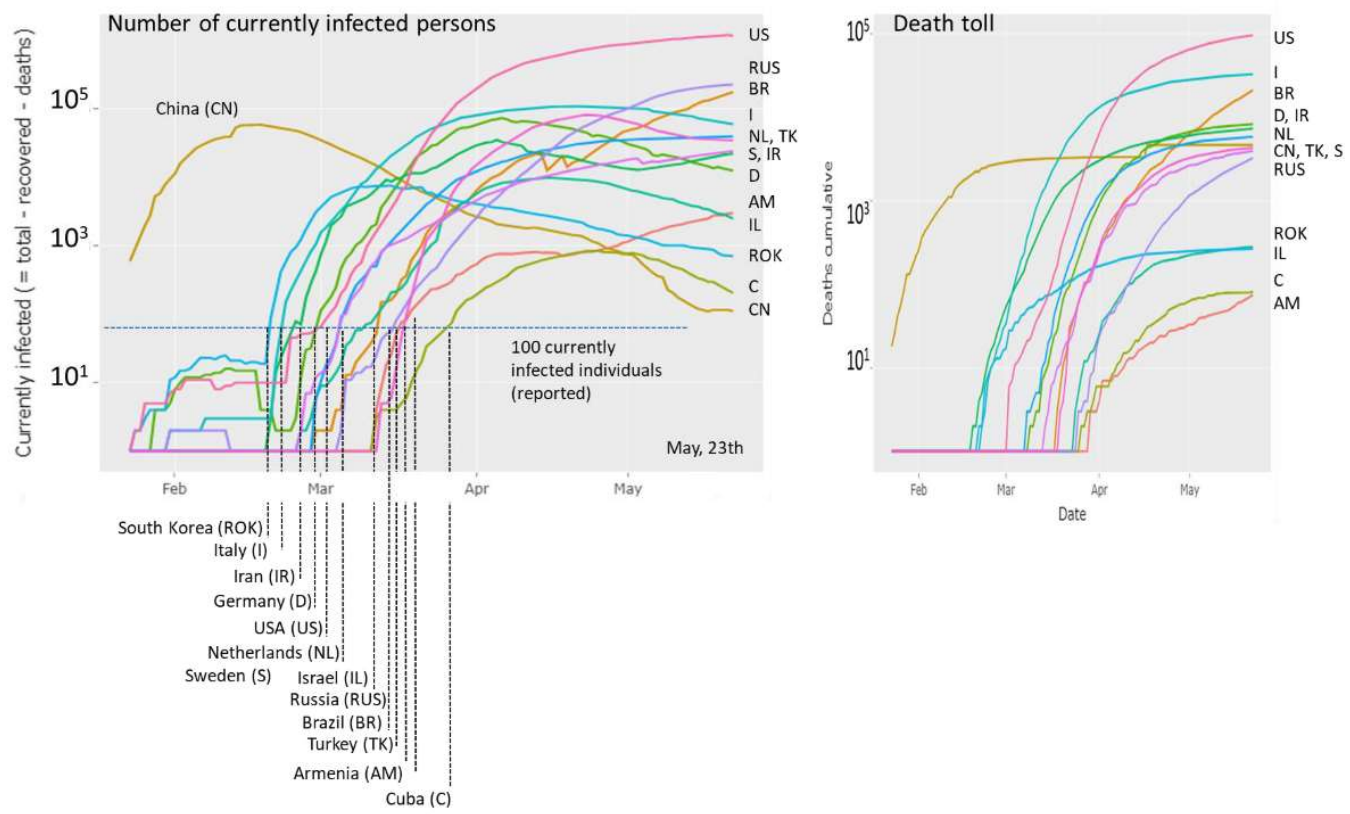

Figure 1. Covid-19 cases (left plot: currently infected, right plot: died individuals) in different countries as a function of date. The '100-cases per country' threshold is crossed between end of February and end of March for the countries shown (except China). The time courses reflect growing (e.g., US, RUS, NL), slightly decaying (e.g., I, D, TK), strongly decaying (e.g., $\mathrm{CH}, \mathrm{ROK}$ ) regimes of epidemic or indications of bi-or multiphasic growth (e.g., AM, IR). The courses of the dead toll as a function of time reflect country-specific percentages of Covid-19 victims. The plots were generated in the Corona-viewer on a daily actualization-basis as described in the text.

Standard epidemic models provide a theoretically well-founded description of dynamics of disease incidence in terms of rate constants for transmission and recovery of Covid-19 and detailed 'generation-interval' and/or 'serial interval' functions [8]. Different models, mostly assuming a series of diseases states such as the 'susceptible-infected-removed' (SIR) types (see below) were used to describe 'epi-curves' of selected countries and regions under consideration of spatial heterogeneous outbreak and transmission scenarios and/or the effect of NPIs [9-26]. Models have been applied not only in a retro-perspective way but also to forecast the epidemic. Because of our still limited knowledge about the disease mechanisms and detailed data about its spread in the population forecasting either provides short-term extrapolations or hypothetical predictions of possible future scenarios as the result of different model assumptions.

We here describe the Covid-19 viewer, a monitoring tool which aims at bridging the temporal 'epi-curve' and the modelling levels. Our monitoring substitute the time-coordinate used in the epi-curves by infected cases (cumulative or current ones). It pursues the phase state trajectory idea suggested previously [27]. The obtained trajectories enable one to visually estimate the dynamic state 
of the epidemic in terms of simple shape characteristics such as slope, parallel shifts or turning points with direct relations to transmission and removal rates of the disease. Comparative analysis between trajectories of different countries enables one to judge different scenarios of NPIs, population size, and social factors. Daily actualized data and interactive web-functionalities enable monitoring the pandemic based on newest data.

The paper is organized as follows: In the Results section we introduce and illustrate the different trajectories and plots available in the monitoring tool by showing examples from different countries of the world, serving as worked examples referring to the state of pandemic in the second half of May 2020 and partly also to the end of June (Supplementary Figures). The majority of plots shown in the publication are taken from the web-tool. The interested reader thus can actualize the data and/or chose countries of interest for similar views. We address the effect of NIPs in Europe, the spread of epidemic in Germany and compare mortalities between selected countries. The Materials and Methods section briefly explains the major functionalities. Details of the methods as well as supplementary figures were provided in the Supplementary File.

\section{Materials and Methods}

\subsection{Tool and Data}

The trajectory-monitoring tool ('Covid-19 viewer') was programmed as web application using the R-package 'shiny' [28]. It processes the number of newly infected and of resolved cases (sum of recovered and deceased individuals) from 187 countries (and of Diamond Princess cruise liner with 712 cases) as provided by the Corona virus resource center of Johns Hopkins Medical University Boston, USA) https: //systems.jhu.edu/research/public-health/ncov/ for the 'World data') and from Robert-Koch-Institut (Berlin, Germany) (https://www.rki.de/DE/Content/InfAZ/N/NeuartigesCoronavirus/Fallzahlen.html) for the case numbers of the German states ('German-country'). Data are daily updated.

\subsection{Availability}

The tool is available via the websites of IZBI (https://www.izbi.uni-leipzig.de/lha/) and the Leipzig Health Atlas (https://www.health-atlas.de/models/28).

\subsection{Functionalities}

The 'Covid-19 viewer' is an interactive tool to monitor the development of the pandemic in 187 countries and in the 16 German states using simple and intuitive plots (Figure 2, Supplementary File). The tool is interactive and enables the user to select different data presentations. The so-called 'rise-fall' trajectory was chosen as 'standard visualization'. It shows the newly confirmed Covid-19 cases per country and per day (averaged over the past 7-days) as a function of accumulated total cases per country in double-logarithmic scale. The 'rise-fall' trajectory typically divides into a 'rising' part of exponential growth part of epidemic and a 'falling' decay regime due to counter measures and/or progressive immunization in the population. It allows estimating transmission and removal rates and reproduction numbers (Supplementary File I). The time range can be chosen and, as an illustration, an animated movie of the rise-fall trajectory for selected countries as a function of time. The user can chose 'custom' trajectories to combine different numbers (infected or removed cases, deaths, daily or cumulative counts, Figure 2) along the coordinate axes for alternative views (use the hoover window for inspecting metadata such as date or numbers). Trajectories can be generated for all countries, groups of countries or single countries. The selection 'worlwide' provides the cumulative numbers of all countries and thus a view on the worldwide status of the pandemic. German states can be selected by choosing 'Germany-state codes'. In addition conventional time series plots show the different numbers (infected, removed, recovered, died as cumulative or per-day) as a function of date. The viewer offers standard browsing functionalities (zooming in and out, image download). 


\section{Country selection: \\ - all (187) \\ group \\ - Single \\ - German states}

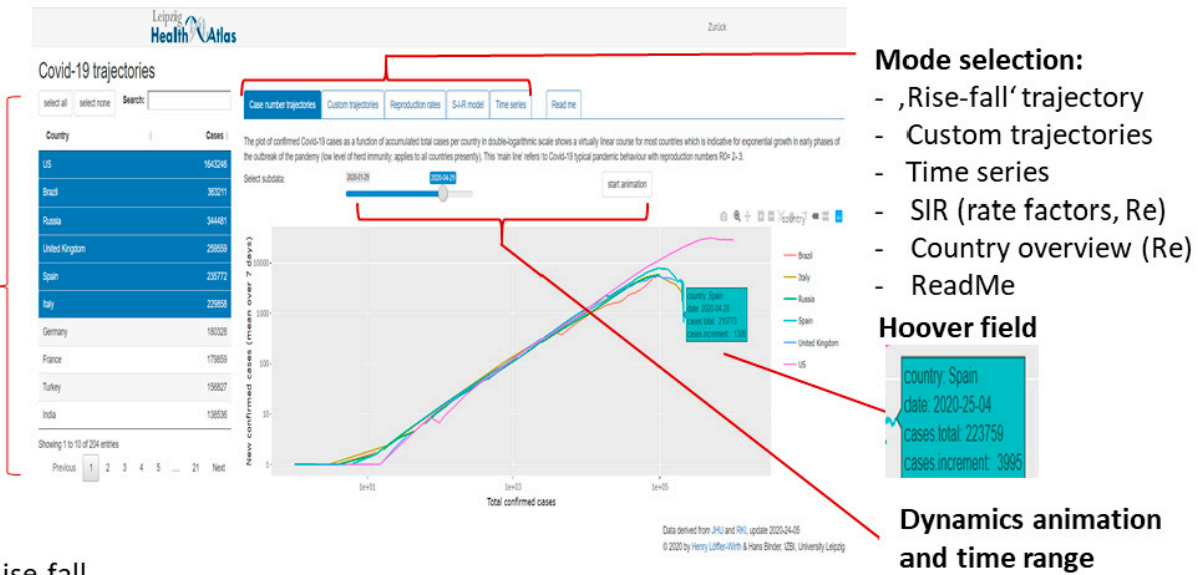

Rise-fall trajectories... of all countries

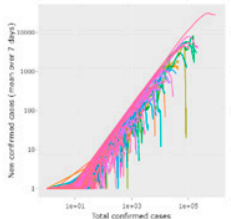

transmission and removal rates

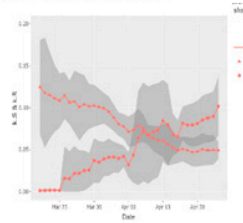

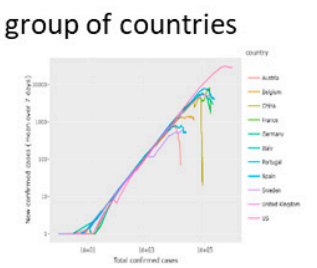

Reproduction number vs time

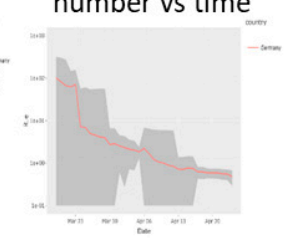

Custom

one country trajectories
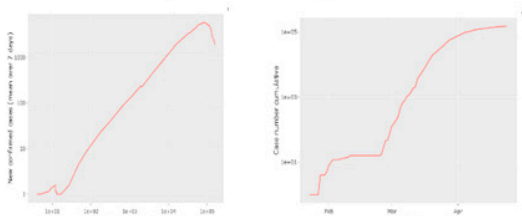

Reproduction number vs countries

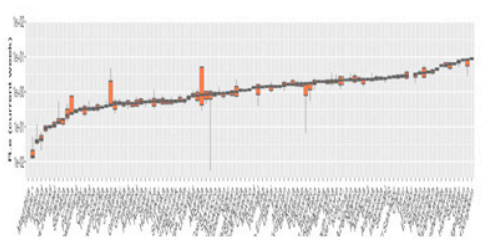

Figure 2. Covid-19 viewer: Screenshot with major functionalities indicated (above) and example plots (below).

\subsection{SIR Model: Parameter Estimation and Visualization}

The susceptible-infected-removed (SIR) model provides a simple, adequate and straightforward interpretation of the data (see Figure 3 for illustration and Supplementary File, Figure S1). It describes the disease as a sequence of three states-S (susceptible), I (infected) and R (removed)—where infection proceeds via interactions between $\mathrm{S}$ and I individuals. Recovered individuals are assumed to get immunized. The respective numbers were reported by census systems, which can differ between countries, e.g., by counting only hospitalized individuals, counting died Covid-19 positive cases as not Covid-19 caused and/or referring to different test-frequencies. All case numbers must therefore be understood as 'visible', i.e. reported ones. True numbers and their relation to testing rates across all countries are provided and discussed recently [29].

The rise-fall trajectories monitor country-specific dynamics of epidemic. Custom trajectories estimate the time-dependent effective transmission and removal rate factors, $c_{e}(t)$ and $k(t)$, respectively. Time courses of the rate factors were extracted from the local slopes of the trajectories (Supplementary File, see also Figure S2). The ratio of the rate factors estimates the effective reproduction number $R_{e}(t)$ defined as the number of individuals who get contaminated by one infected person on the average. The time-dependent rate factors depend on the intrinsic properties of Covid-19 and on a series of external factors such as Public Health Measures (non-pharmaceutic interventions, NPIs) to slow down transmission of epidemics (affecting $c_{\mathrm{e}}$ ) and effective medical services after infection (affecting $\mathrm{k}$ ). 

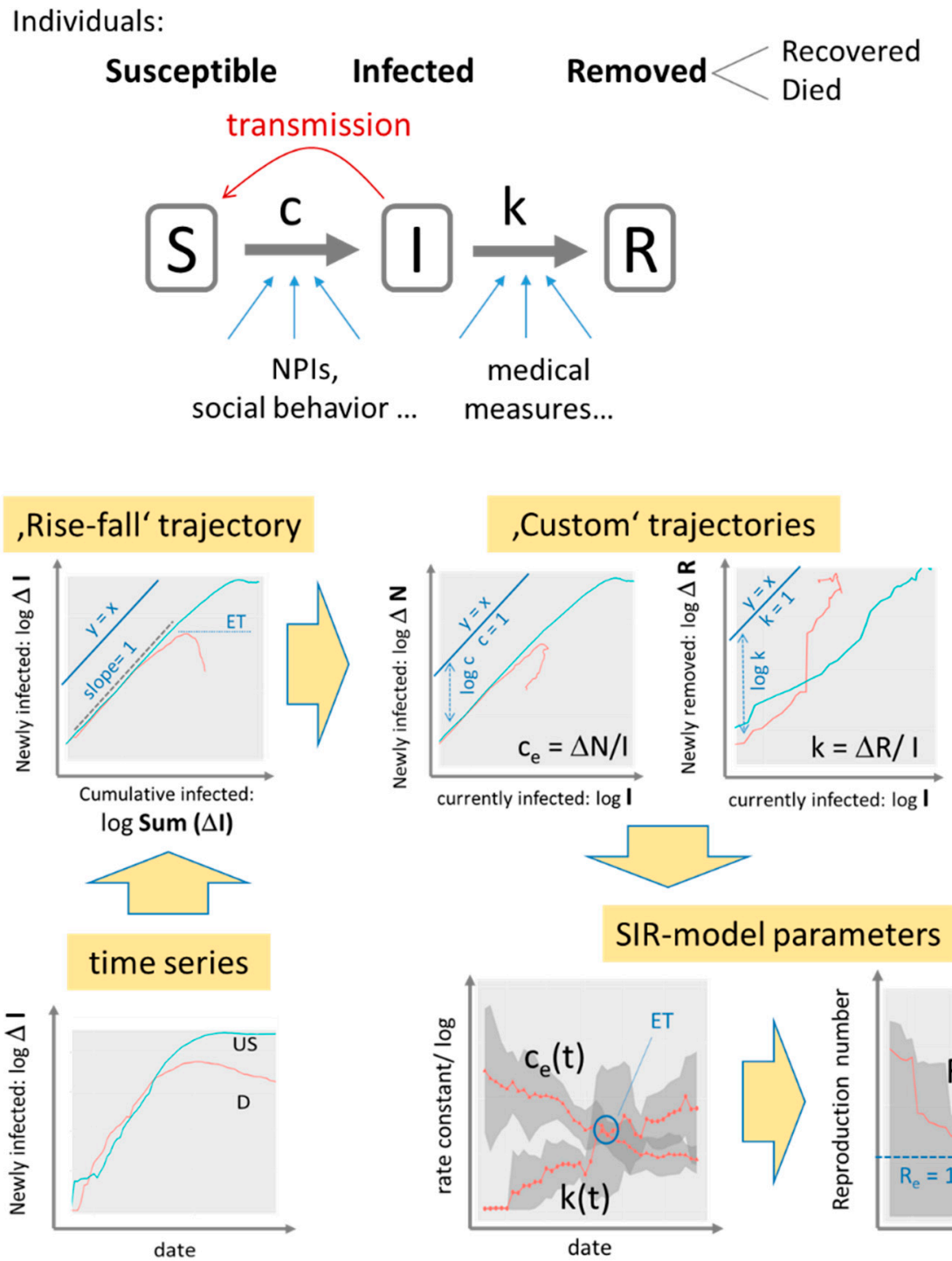

\section{SIR-model parameters}
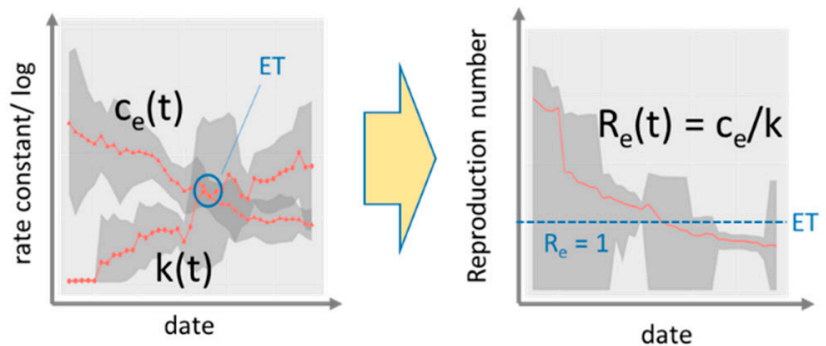

Figure 3. SIR model analysis: The SIR model assumes a sequence of three disease states (S, I, R, $\mathrm{S}+\mathrm{I}+\mathrm{R}=\mathrm{N}, \mathrm{N}$ population size) and transitions between them. Transmission and removal rates (numbers per day) are quantified in terms of the rate constants $\mathrm{c}$ and $\mathrm{k}$, respectively. The time series of infected cases transforms into trajectories, which show daily changes of infected and removed cases as a function of cumulated or current infections in double logarithmic scale. Exponential growth is indicated by slope of unity while the transmission and removal rate factors refer to the vertical distance between the respective trajectory and the diagonal line $(\mathrm{y}=\mathrm{x})$. A downwards curved trajectory indicates decreased growth or eradication of epidemic (slope $<0$ ) with the epidemic threshold (ET: slope $=0$ ) indicating stop of epidemic. Values of the factors ce and $\mathrm{k}$ and of the reproduction number Re are plotted as a function of date as running averages over a sliding window of seven days (the running standard deviation is shown as grey uncertainty strip). Example data are shown for Covid-19 case numbers in Germany (D, red curves) and USA (US, blue) from 27 April 2020. See text and Supplementary File, Appendix I and II for details.

In addition to the estimation of SIR parameters as described above, we performed least-squared fits of the trajectories where the daily numbers of newly infected and removed cases were calculated as a function of the cumulative number as predicted by the SIR model to estimate $\mathrm{N}_{\max }$, the maximum cumulative number of infected cases (Supplementary File, Figure S1). 


\section{Results}

\subsection{Monitoring the State of Epidemic Using 'Rise-Fall' Trajectories}

The 'rise-fall' trajectory plots the newly confirmed Covid-19 cases (averaged over running 7-day windows) as a function of accumulated total cases per country on a double-logarithmic scale. This presentation is equivalent to phase space plots commonly used to represent dynamical systems in physics. While data along the trajectory are 'dynamic', i.e. they refer to a sequence of days from left to the right, the time does not explicitly appear. Such a plot enables a direct comparison of the otherwise nonsynchronous curves of the disease progression for the different countries [27]. The 'select all' function shows the trajectories of all countries considered (Figure 4a). Overall, the double -logarithmic trajectories reveal two basic features: An initially linear increase with a slope of unity indicates exponential growth of epidemic. This linear regime is followed for many countries by a downwards turn which indicates slowing down of growth owing to NPIs 'locking down' infections and/or possibly also to progressing immunization of the population in later phases of epidemic leading to the depletion of the reservoir of susceptible individuals and/or other factors.

The 'rise-fall' plots use the cumulative number of cases $\mathrm{N}$ as a robust measure of progressing epidemic in a population. Naturally, it is larger for countries with larger population sizes providing a larger overall reservoir for Covid-19 infections compared with smaller countries. The shapes of the 'rise-fall' trajectories are however virtually independent of country size. The trajectories thus reflect intrinsic properties of epidemic in terms of its transmission and removal potential. The two sets of trajectories shown in the left and right part of Figure 4a refer to situation on 17 April 2020 (see also Figure S4) and about six weeks later, respectively. For most countries, among them France, Italy, Spain and Germany, the trajectories turn into falling courses during this time and/or the falling parts further drop and intersect the '0.01 -slope' line referring to a more than tenfold reduction of the transmission rate of epidemic (see below). These trends thus indicate decay of pandemic after the NPIs taken in most of countries. On the other hand, Brazil and Russia emerged as the countries with most cumulative cases after the USA, with still growing case numbers. At the time of the last revision of this manuscript (8 July) India and Latin American countries (Mexico, Chile, Peru) had replaced Russia thus indicating the highly dynamic nature of Covid-19 pandemic.

Hence, the 'rise-fall' trajectories illustrate the current state of the epidemic and its developmental course with country-wise resolution. They enable monitoring the state in terms of differences and similarities between the countries and geographic regions revealing specifics and commons of epidemic spread: (i) A unique linear slope of most of the trajectories in the intermediate abscissa range is indicative for exponential growth in early phases of the outbreak of the pandemic (low level of immunity in the population). The nearly identical position of these lines refers to Covid-19 typical pandemic spread rate and maximum basic reproduction numbers $\mathrm{R}_{0}$ (Supplementary File). (ii) Parallel, down-shifted but increasing lines suggest still exponential growth, however with reduced rates reflecting reduced effective reproduction numbers $1<\mathrm{R}_{\mathrm{e}}<\mathrm{R}_{0}$. In these countries (e.g., Sweden, Iran), the epidemic is not stopped. (iii) The 'flattening' of slope and downwards curvature seen, e.g., for most European countries such as Italy, Spain or Germany reflects slowing down of growth owing to efficiency of NPIs and, possibly also progressive immunization in the population. (iv) The sharp, virtually vertical drop of trajectories reflects the stop of an observed epidemic, e.g., for China and South Korea, and after May 1st for New Zealand, Australia, and also several European countries, where about one month later, indications of a second wave of epidemic growth become evident by again sharply increasing trajectories (see below). (v) The different qualitative features of the trajectories are virtually independent of population size of the countries. 'Smaller' countries like Iceland, Cyprus, Armenia or Georgia show overall similar features such as linear rise, parallel shifts (Armenia), a maximum and steep falling parts (e.g., Iceland). 


\section{a) Overview: all countries}
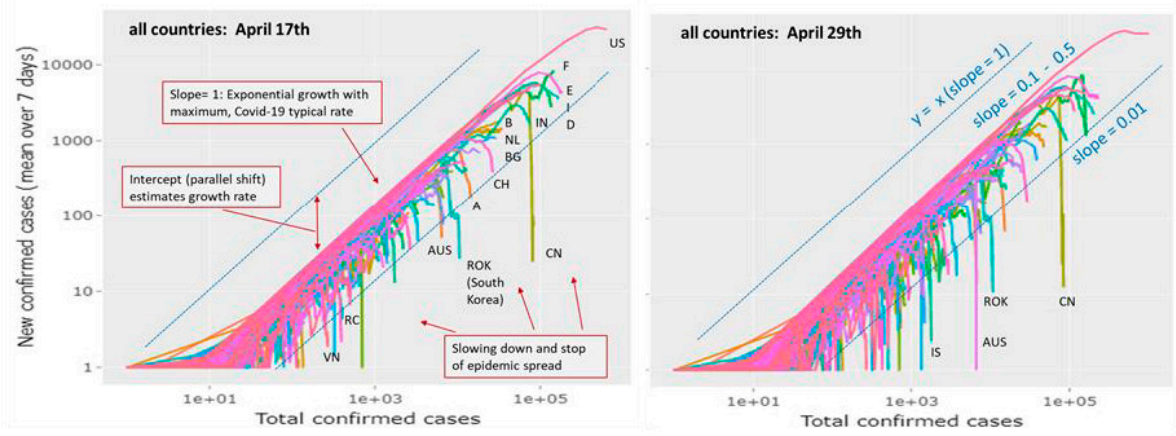

\section{b) Groups of countries selected by size and region}
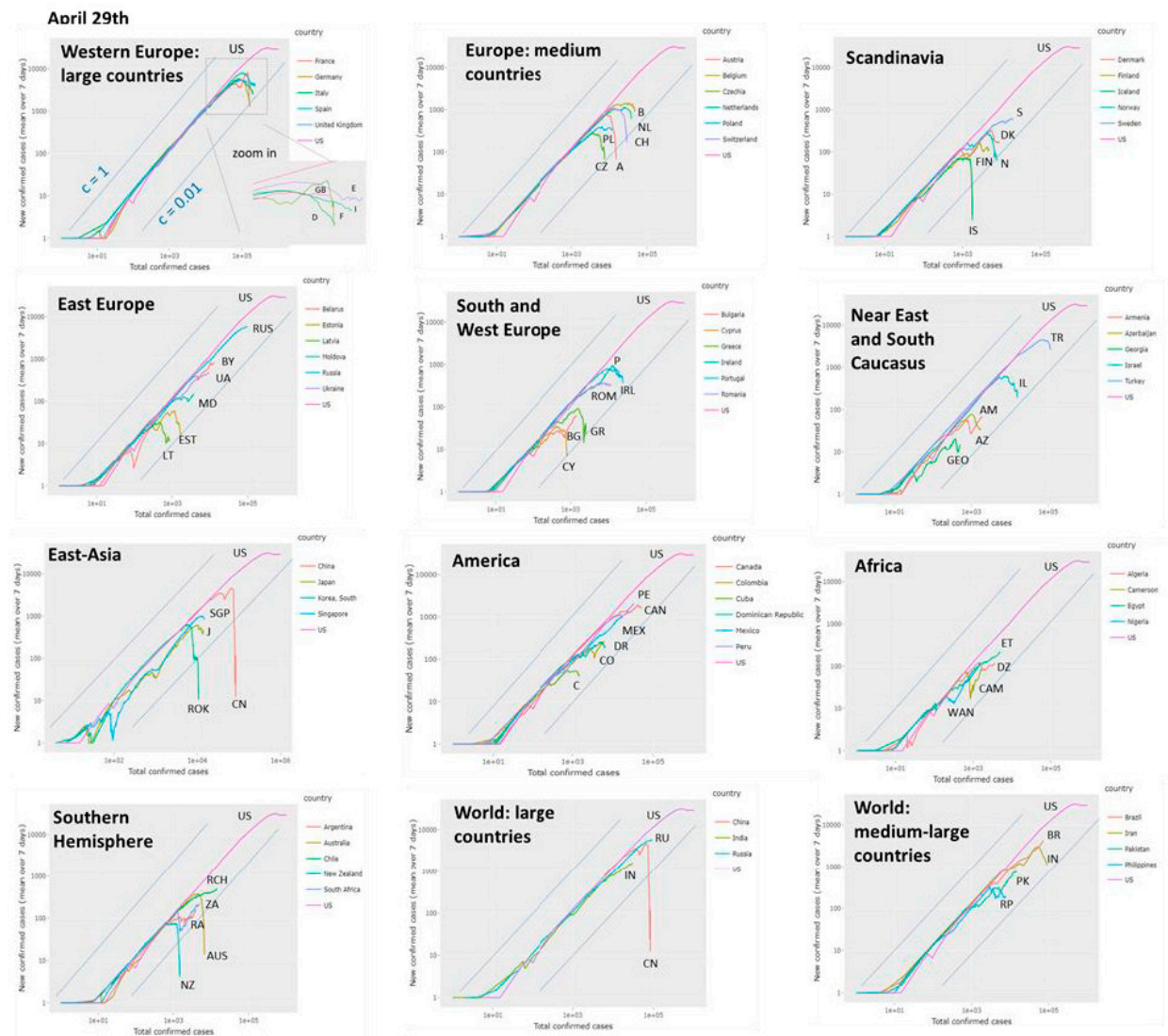

Figure 4. 'Rise-and-fall' trajectories of Covid-19 pandemic: (a) Trajectories of all 187 countries considered by Johns Hopkins Data Center plot new confirmed cases as a function of total cases in double logarithmic scale. They visualize the state of the epidemic with country-wise resolution. Exponential growth is reflected by a line parallel to the diagonal $y=x$ (slope $=1)$ followed by a fall reflecting slowing down of the epidemic. The slope equals the difference between the transmission and removal rate factors, $\mathrm{c}-\mathrm{k}$. The slope $=0.01$ line consequently refers to the more as tenfold reduction of the maximum transmission rate observed for many countries in their linear rising part. With time (compare left and right plots) the number of countries crossing this line increases. (b) Trajectories for groups of countries characterize the epidemic in countries of different population sizes and regions of the world. Trajectory of the USA (with the highest infection numbers worldwide) is shown for comparison in all plots. Letter codes for countries were chosen according to the international vehicle registration code (https://en.wikipedia.org/wiki/International_vehicle_registration_code). 


\subsection{Monitoring Country-and Worldwide Situation of Pandemic}

Detailed inspection of the trajectories of sets of countries grouped roughly by size and geographic region reveals further details (Figure 4b, compare with Figure S4 in Supplementary File showing the respective trajectories recorded at 29 April). Most Western European countries of larger and medium size reached the decaying part in the first week of April 2020 (except Sweden and Great Britain) roughly two-three weeks after NPIs were taken in these countries. Countries from different parts of the world such as Austria, Iceland, South Korea, Australia, New Zealand and China reached low levels of new infections as indicated by strong vertical decays. Larger countries (e.g., Russia, India, Brazil, Pakistan, India) were in the rising part. Some countries show a two-phase growth as indicated by the parallel right shift of linear regions in their growing part (e.g., Sweden, Denmark, Iran, Ukraine, Armenia) indicating that fast exponential growths are followed by slower phases due to reduced transmission rates (see below). Singapore and Japan show relatively slow growth phases with reduced rates and late turns into falling regimes while South Korea's turn is very sharp, presumably because of the 'crash down' measures taken there.

According to different regions and country sizes one finds that larger European countries (D, F, E, I, we use vehicle country codes for abbreviations of countries) except GB, selected middle-sized European countries (B, NL, CZ, CH, A) except PL and Scandinavian countries (DK, N, FIN, ISL) except $\mathrm{S}$ show a strong drop of epidemic. The exceptions are characterized by slowed-down growth (S, PL, GB). East Europe (RUS, UA, BY, MD), South Caucasus (AM, GEO, AZ), selected American (US, $\mathrm{PE}, \mathrm{MEX}, \mathrm{CO}, \mathrm{DR}$ ) and African (ET, WAN, CAM, DZ) countries all did not reach the turning point into the falling regime. Exception from these regions are CAN, C, the Baltic countries (EST, LAT), TK and IL. States from the Southern Hemisphere split into ones with stopped epidemics (NZ, AUS) and ones with still spreading epidemics (RA, ZA, RCH), also seen for large (IND) and medium-sized countries (BR, PK, IR). Overall inspection of trajectories indicates that the spreading pandemic moved from East Asia and the Middle East and West Europe towards South America, South Asia and Africa. South Caucasus, Middle Asia (IR) and Eastern Europe show a heterogeneous picture.

The cumulative number of cases of all countries considered shows a still rising tendency until the end of June which clearly shows that pandemic is far from being stopped (Figure S5). Countries like US, Brazil and India are presently among the Covid-19 drivers. The rise-fall trajectory further enables stratification of countrywise-state of epidemic in countries that 'beat Covid-19', that 'are nearly there' and that 'need to take actions' according to https://www.endcoronavirus.org/countries (Figure S6).

\subsection{Monitoring Covid-19 by Custom Trajectories, Epi-Curves and Rate Factors}

The 'rise-fall' trajectory uses cumulative cases $\mathrm{N}$ along the abscissa as a robust measure of the extent of the epidemic. These numbers don't consider the degree of recovery and thus they don't reflect the current amount of infected cases (I). Custom trajectories make use of the independent number of removed cases $(\mathrm{R})$ reported and plot cumulative, current and differential (per day) numbers in different combinations (Supplementary File). Using the current number of infected cases $(I=N-R)$ as $x$-axis (in log-scale) one sees whether the extent of infection increases or it decays. While the rise-fall trajectory, $\Delta \mathrm{N}-\mathrm{vs}-\mathrm{N}$, tends asymptotically towards a maximum cumulative number of infections $\left(\mathrm{N}_{\max }\right)$ for each country, which reached the falling regime, the $\Delta \mathrm{N}-\mathrm{vs}-\mathrm{I}$ trajectory turns from a growing I into a decaying branch at $\mathrm{I}_{\max }$, the maximum number of infected individuals. These trajectories turn in clockwise directions for most countries meaning that the rate factor of transmission of epidemic, $c_{e}(t)$, decays (Supplementary File). For example, Austria and Japan show full turns while the turns of Sweden and USA remain incomplete leading to less pronounced decays of the respective $c_{e}(t)$-courses (Figure $5 \mathrm{a}, \mathrm{b}$ ). In contrast, the $\Delta \mathrm{R}$-vs-I trajectories turn typically in counterclockwise direction indicating an increase of the removal rate factor as explicitly seen in the respective $\mathrm{k}(\mathrm{t})$-plots. The ratio of the effective transmission and of the removal rates then estimates the effective reproduction number as a function of time, $R_{e}(t)$ (Figure $5 b$ ). The trajectories of the countries selected for illustration reflect different trends such as strong and straight repression and stop of epidemic via reduction of 
transmission in Austria, reduced growth but still expanding epidemic in USA and Sweden as well as indications of a second wave of expanding epidemic in Iran. Here, the respective trajectories and plots of rate factors and of $R_{e}(t)$ show different aspects of the dynamics of the epidemic. For example, Sweden and Japan are characterized by relatively low levels of rate factors compared with Austria and Iran, a difference seen also in the parallel shifts of the respective trajectories.

\section{a) Trajectories and ,epicurves'}

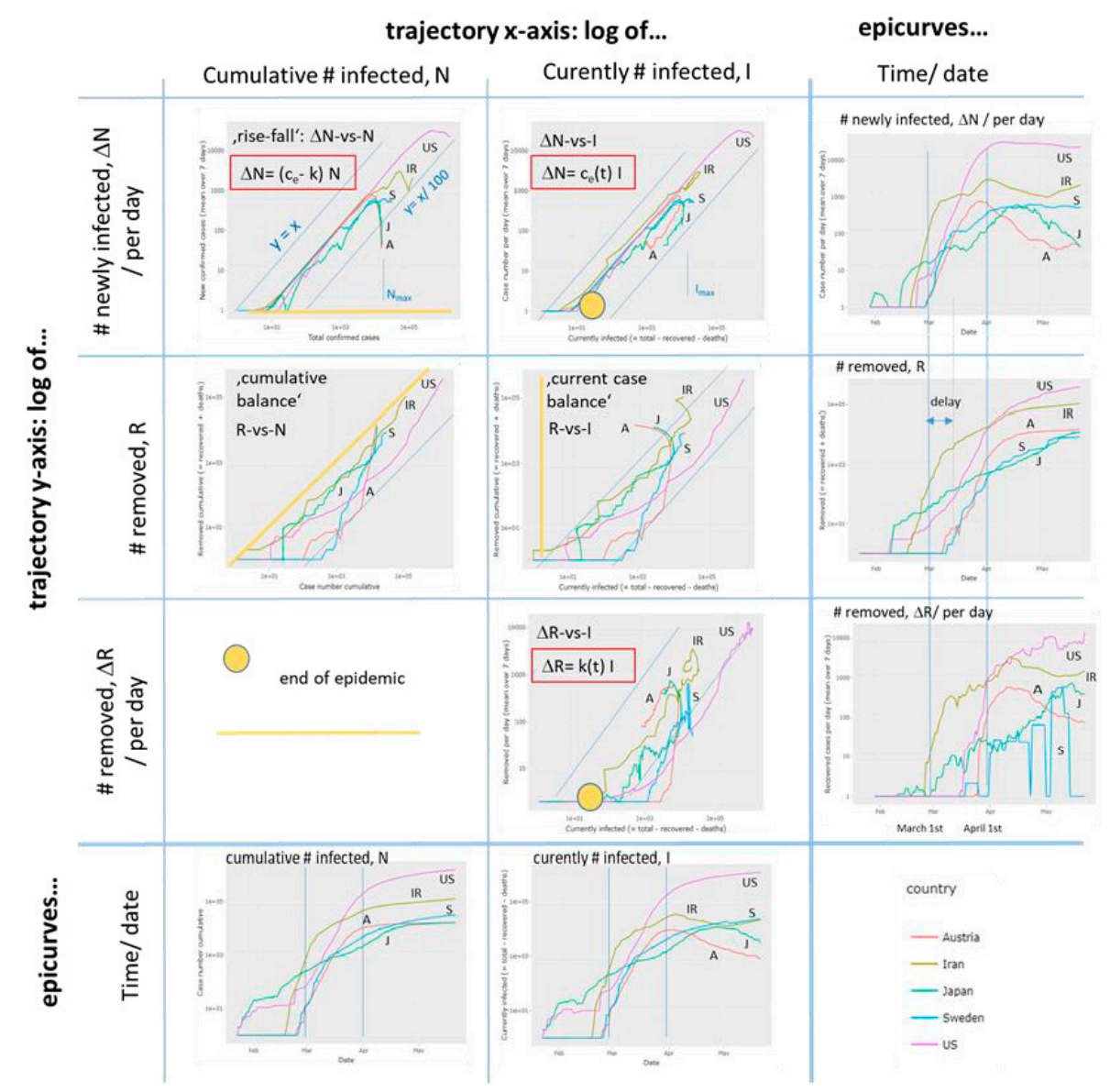

\section{b) Rate factors and reproduction number}

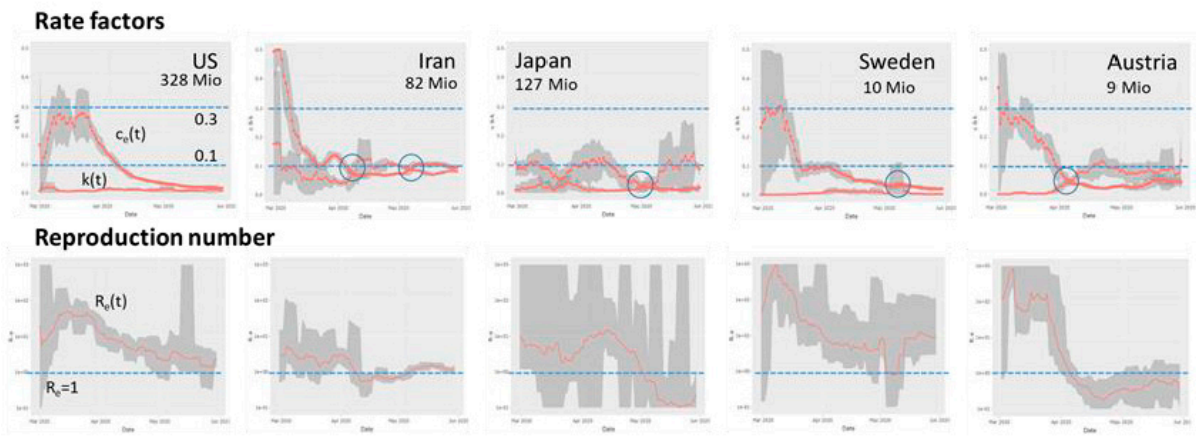

Figure 5. Dynamic Covid-19 characteristics of selected countries. (a) Trajectories and the respective time dependencies ('epicurves') plot different numbers as indicated in the figure. The yellow marks (circle and lines) indicate the 'end' points where the trajectories are expected to return upon eradication. See also Supplementary File for details. (b) Rate factors of transmission and the respective reproduction numbers as a function of data. 
In Supplementary File we can find analogous differences between Western and South European countries (E, F, I, Figure S3) compared with Middle European ones (D, A, CH), which suggest differences in the spread mechanism of Covid-19 and, possibly, also in the recovery dynamics. The removal rate obtained depends on the time-delay between infection and recovery [30], which is neglected in our simple trajectory-approach (see also epicurves in Figure 5a). 'Cumulative balance' and 'current case' custom trajectories complete the visualization options: Lower levels of transmission and removal rates associate with trajectories running closer to the diagonal. Overall, the trajectories enable tracing an epidemic in terms of case numbers reported directly be the census agencies of the respective countries. Derived numbers such as the rate factors and reproduction numbers 'translate' these numbers into features more directly describing the dynamics of the epidemic.

\subsection{Monitoring Covid-19 by Reproduction Numbers}

Effective reproduction numbers as shown in Figure $5 \mathrm{~b}$ provide suitable summary measures of the case numbers with a well-defined epidemiological meaning. Their value defines the transmission potential in the population in terms of the mean number of individuals who are infected by one infectious person on the average. For the comparison of all or a selection of the countries, the monitoring tool generates a ranked boxplot of reproduction numbers. In roughly $50 \%$ of all countries $R_{e}$ is still larger than unity meaning that epidemic is not stopped (Figure 6).

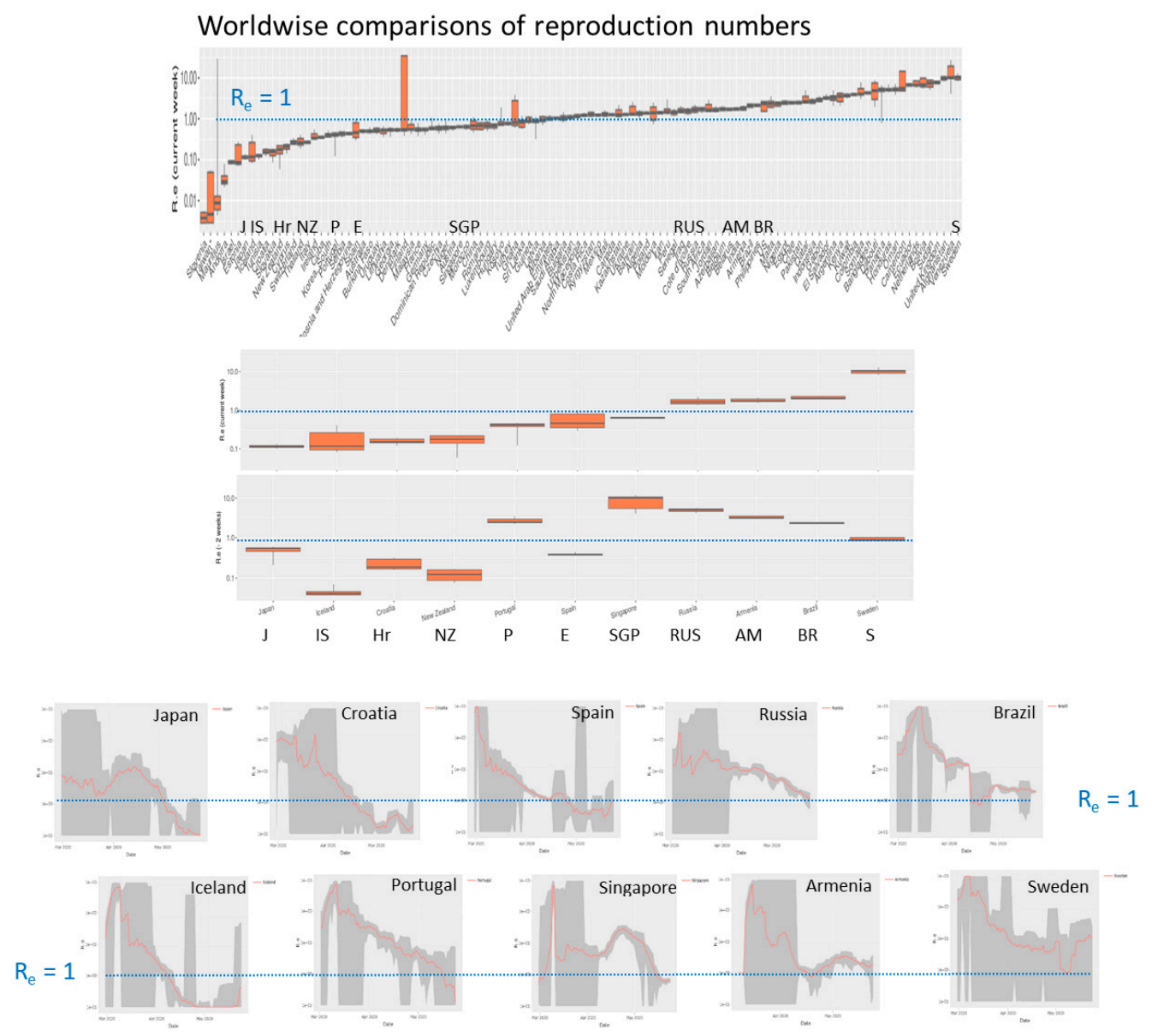

Figure 6. Effective reproduction numbers across the world. Ranked plot of current Re values across all countries studied indicate that less than half of them fall below the epidemic threshold of $R_{e}=1$ (horizontal dashed line). Time series for selected countries are shown as examples in the part below. They reveal sharp decays, virtually invariant and even increasing courses. 
The tool also generates the respective plot for $\mathrm{R}_{\mathrm{e}}$-values obtained two and four weeks before when about $70 \%$ of countries show $R_{e}>1$, which demonstrated a decaying trend. $R_{e}$-time courses of selected countries illustrate different types of decays, which presumably relate to the NPIs taken. For example, early, consequent eradication of epidemic in Island and Croatia result in fast and steep decays. Slower but monotonous decays were observed in Russia, Spain and Portugal. Wave-like changes before the final decay (Japan, Singapore) or even worsening of the situation (Armenia, Sweden until the middle of May) were also found. Presently (25 May) Sweden shows the highest reproduction numbers among all countries studied. Note, that $R_{\mathrm{e}}$ is a relative measure considering daily changes and current numbers of infections and recoveries (Supplementary File I), meaning that restricted outbreak clusters affecting only relatively small numbers of individuals can suggest spread of epidemic in a larger, not affected population. Hence, a combination of different number characteristics should be used to characterize the dynamics of epidemic, namely the transmission rate factor (or doubling time of cases) especially at the beginning of epidemic, effective reproduction numbers more in the phase of vast spread and absolute numbers of new cases in the phase of mitigation and near eradication. The trajectories visualize all these different features at once.

\subsection{Monitoring the Effect of NPIs Across Europe}

The NPIs were taken in Middle and Western Europe and Scandinavia in the first three weeks of March 2020. Next, we asked how these measures affected the dynamics of the epidemic. The rise-fall trajectories of countries selected from $[12,31]$ reveal that they now are mostly in the falling regime however with modifications such as parallel downwards shifts, wave-like decays and even lacking decays as already discussed above (Figure 7a). In Figure $7 \mathrm{~b}$ we replot the trajectories separately for each country together with marks indicating which measure was taken when along the trajectories. In most cases, trajectories start turning downwards about two weeks after a complete lockdown in the respective country. Before this, one often finds slowing down of the exponential growth as indicated by small differences compared with the trajectory of US referring to exponential growth. In Norway, Denmark, and also Sweden one observes a relatively strong first slowing down as indicated by the parallel downwards shift of the trajectories, which roughly refers to a reduction of the transmission rate constant by about $30 \%$ (Figure $7 \mathrm{~d}$ ). Sweden, without complete lockdown measures, but also Great Britain show the weakest decay of the trajectories and largest values of the effective reproduction numbers Re $>1$ in contrast to all other countries except Belgium (Figure 7c). Comparison of the reproduction numbers two and four weeks earlier indicates high values in Britain and Sweden and also a delayed decay in Italy, Spain and France. These countries were heavily hit by Covid-19 in February and March. The time courses of the reproduction numbers $\operatorname{Re}(t)$ respond on the measures in many cases showing, at least, small drops in support of a recent study [12], which assumes that the reproductive number-a measure of transmission-immediately responds to interventions being implemented (Figure 7d, the first measure and complete lockdown were indicated). Consistent decays to values Re $<1$ after about two weeks were seen in Scandinavia (except Sweden) and Austria, Switzerland and Germany while in Belgium, France, Italy and Spain the decays last roughly four weeks until they fall below the epidemic threshold. In Sweden and Great Britain, virtually unchanged levels of Re above the ET were observed. A recent model analysis of the effect of NPIs in Germany applies a SIR model with changed rate constants at so-called 'change points' which are assumed to take place when measures were applied [32]. School closures ('sc') were found as most effective in reducing reproduction numbers by about $50 \%$, where 'sc' took place shortly before complete lock down in most countries [33]. We found a decay of the transmission rate by $60-70 \%$ during the time when measures were applied in rough agreement with [32] (Figure 7d, right part). Another recent reanalysis of the NPI-data across Europe used in [31] found that the different NPIs were introduced so closely in time during some weeks in March that makes identifiability of the effect of individual NPIs infeasible [34]. Interestingly, Japan showed a similar response to NPIs as the European countries. Namely a slow, but instantaneous 
growth of epidemic turned into the falling regime at the beginning of May (Figure 5 and Figure S7), two-three weeks after NPI measures were intensified at 10 April.
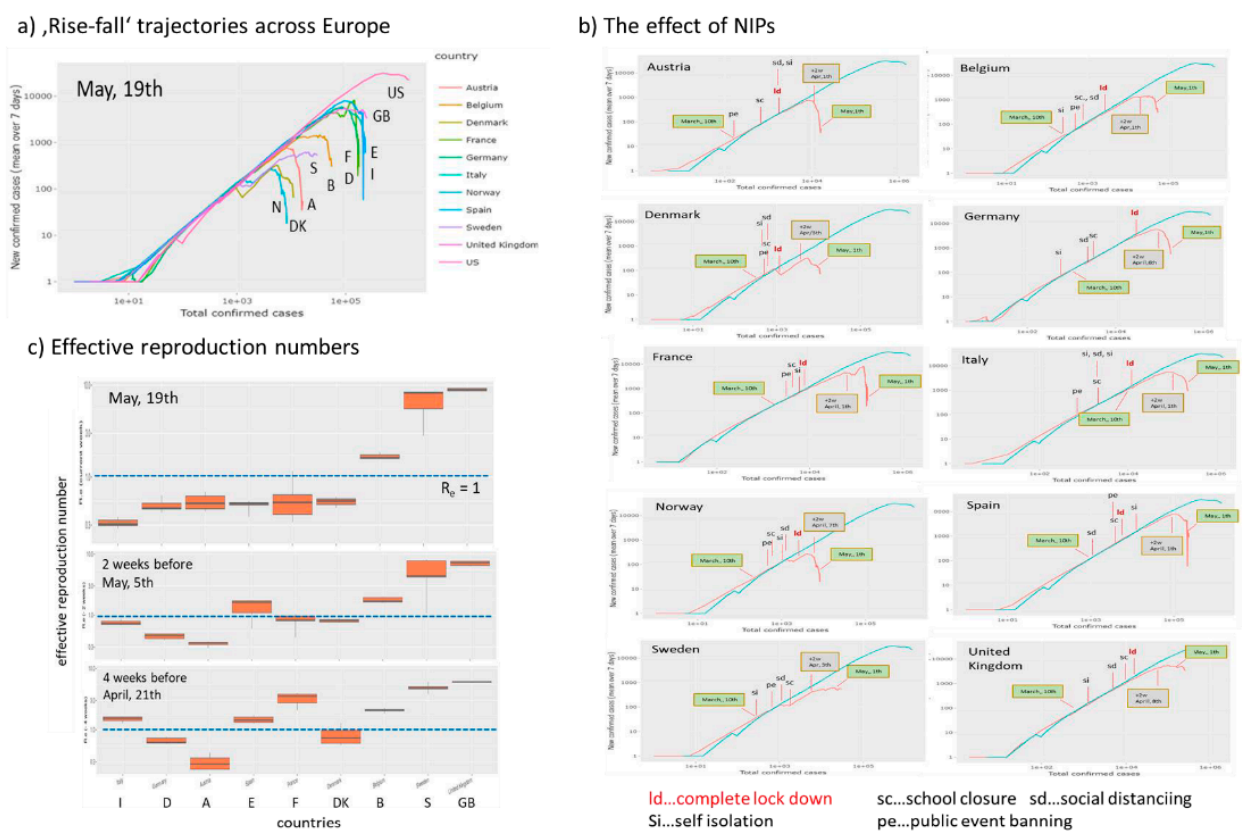

d) Time courses of the effective reproduction numbers

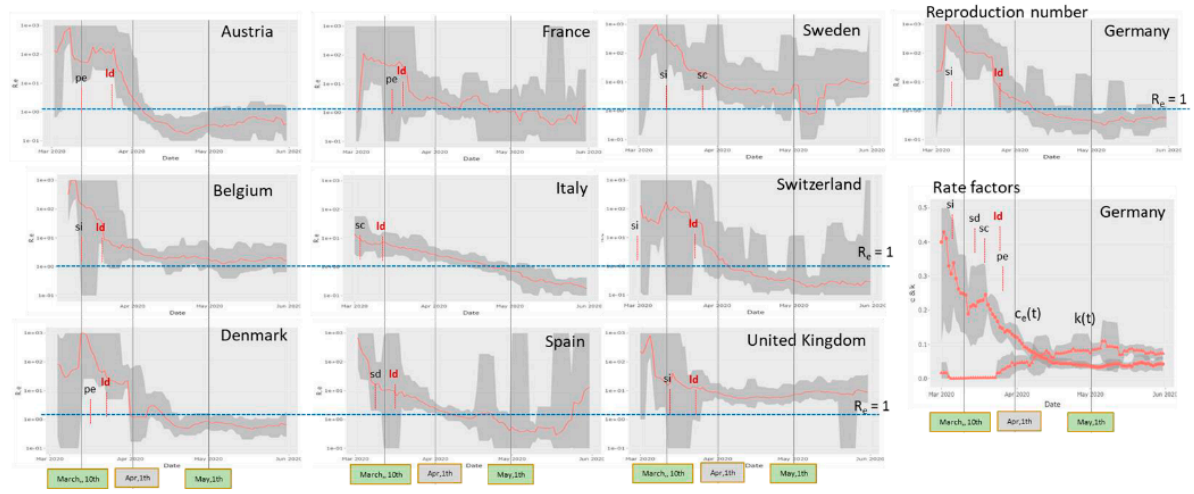

Figure 7. The effect of Non-Pharmaceutical Interventions across ten European countries selected in analogy with [12]. The trajectory of US is shown for comparison. (a) Rise-fall trajectories of selected countries mostly decay thus indicating marked decrease of epidemic in most cases. (b) Country-by-country plots of the rise-fall trajectories together with marks assigning the NIPs (dates and assignments were taken from [12]) show that the trajectories turn downwards about two weeks after lockdown in most cases (the grey box refers to the 'two weeks after the last measure' date). Exceptions are Sweden (no complete lockdown) and United Kingdom. The two green boxes indicate the data obtained at 10 March (mostly before measures) and 1 May. (c) The effective reproduction numbers are still clearly above the critical value of $R_{e}=1$ for Sweden and Great Britain. Italy and France show the strongest decay of Re over the last 4 weeks. (d) Courses of the effective reproduction number as a function of time indicate a marked drop of $\operatorname{Re}(t)$ immediately after the lock down in most countries. Also the first measure taken is indicated. For Germany, all five measures were indicated together with the courses of the rate factors.

Overall, our simple analysis reveals that NPIs were followed by drops of the reproduction number mainly due to a decay of the transmission rate factor and by halt of epidemic after two to four weeks after complete lock down. Sweden (and partly GB) shows also a drop of $R_{e}$ and of the transmission rate, which however overall are insufficient to stop epidemic until end of May. Both, the time-course of 
reproduction number and the rise-fall trajectory are sensitive to detect the slowing down and the halt of epidemic. The available country-wise numbers used do not allow to analyze the observed effect assuming heterogeneous effects of NPI on different subpopulations, which, in principle, could explain steps and wavelike changes in the courses of the trajectories as an alternative to alterations of the rate factors in a homogeneous population assumed here.

\subsection{Monitoring Covid-19 Across Germany}

Countries across the world differ in many factors related to Covid-19 epidemic such as the particular NPI measures, social behavior, family structure and education systems with differing school rules, population densities, urban structure, transport system and also age distributions and incidences of diseases of increased risks for Covid-19 infections such as obesity, diabetes, renal and cardiovascular diseases. The heterogeneity of these factors is assumed to be smaller inside each of the countries than between them. For Germany, the Covid-19 viewer provides 'rise-fall' trajectories for all sixteen German states, which refer to population sizes between about 0.7 Mio (Bremen) up to 18 Mio inhabitants (Nordrhein-Westfalen). They include three city-states (Hamburg, Bremen, Berlin), while the other states are 'area'-states including countryside regions and towns of different sizes. The trajectories overall express very similar courses of the epidemic across Germany (Figure 8a), which suggest relatively similar dynamics of the epidemic in different parts of the country. Moreover, Germany-wide NPIs obviously 'locked-down' epidemics in the different states in a similar way. Analysis of the maximum cumulative number of infected individuals, $\mathrm{N}_{\max }$, using fits of the 'rise-fall' trajectories however reveals considerable differences especially between the South and West of Germany and its East and North (Figure 8b).

In Bavaria, which is located in the South of Germany, roughly eight-times more people are infected on relative scale than in Mecklenburg-Vorpommern located in the North-East. In general, 'area' states from the West and South of Germany were more affected by epidemic than states in the East and North. This difference associates with an earlier outbreak in the former states with higher amounts of infected individuals (Figure 8c). NPIs were taken Germany-wide at the same time between 9 and 23 March, which suggests that delayed measures with respect to the outbreak will increase the burden of infections. In summary, Germany-wide the trajectories reflect similar dynamics of epidemic where however earlier outbreaks especially in the West and South and in larger cities give rise to increased numbers of infected persons, possibly because of the relative delay of NPI.

\subsection{Monitoring Death Tolls}

Mortality is an important endpoint of Covid-19 epidemic related to a series of factors such as the intrinsic severity of the virus [35] in first instance, but also age, sex, genetic and immunological predisposition [36], disease history and also co-morbidities of the patients [37], as well as the effectivity of medical measures such as ICU services [38], the capacity of health care systems and also socio-economic factors.

Censing of deaths, e.g., by counting Covid-19 positively tested deaths as Covid-19 related ones, is another factor affecting the reported numbers [29]. So far we subsumed the numbers of death cases together with recovered individuals as removed ones. Separate counting shows that, overall, the dead toll of Covid-19 ranges from less than $1 \%$ up to more than $10 \%$ of counted infections, depending on country and time, when the data were registered (see below). The 'custom trajectory' page provides 'mortality trajectories' in terms of cumulative death cases versus cumulative infections (alternatively one can choose daily cases). Constant percentages refer to parallel diagonal lines as indicated by 'iso-percentage' lines in Figure 9. We selected groups of countries for comparison with part of the 'rise-fall' trajectories in Figure 4b. Larger-sized West-European countries (Great Britain, France, Spain, Italy) all show similar mortality trajectories referring to about $10 \%$ of the (visible) infected individuals. Mortality of Germany and Austria is smaller (about $4 \%$ ), possibly due to the younger mean age of infected persons at the beginning of epidemic. The respective mortality-trajectories however slowly 
grow in direction of the level of the other European countries with increasing number of infections reflecting that increasing age of infected persons. Note that the slopes of the trajectories of the latter countries (e.g., France, Italy, Spain) is slightly steeper than that of the iso-percentage lines which indicates slowly growing mortalities across in these countries. Presently, mortality in Europe is largest in Sweden, Belgium (presumably due to conservative death definition including unconfirmed Covid-19 cases [29]), Netherlands and Great Britain with further increasing trends. Relative small mortality is found in Russia and Belarus possibly because of governmental control of the Covid-19-related death census data [29]. Mortalities in Ukraine and Estonia and in East Asia (China, Japan) are comparable with mortality in US, where the latter Asian countries and also South Korea show an increasing mortality. In South America, one finds higher mortalities than in the US, with further increasing trends. Overall, comparison of the mortality trajectories reveals systematic differences, which need further analysis for interpretation, e.g., by considering statistics of excess deaths. Presently, differences between more limited and more conservative death definitions in different countries impact death estimates [29].

a) ,Rise-fall' trajectories across German states

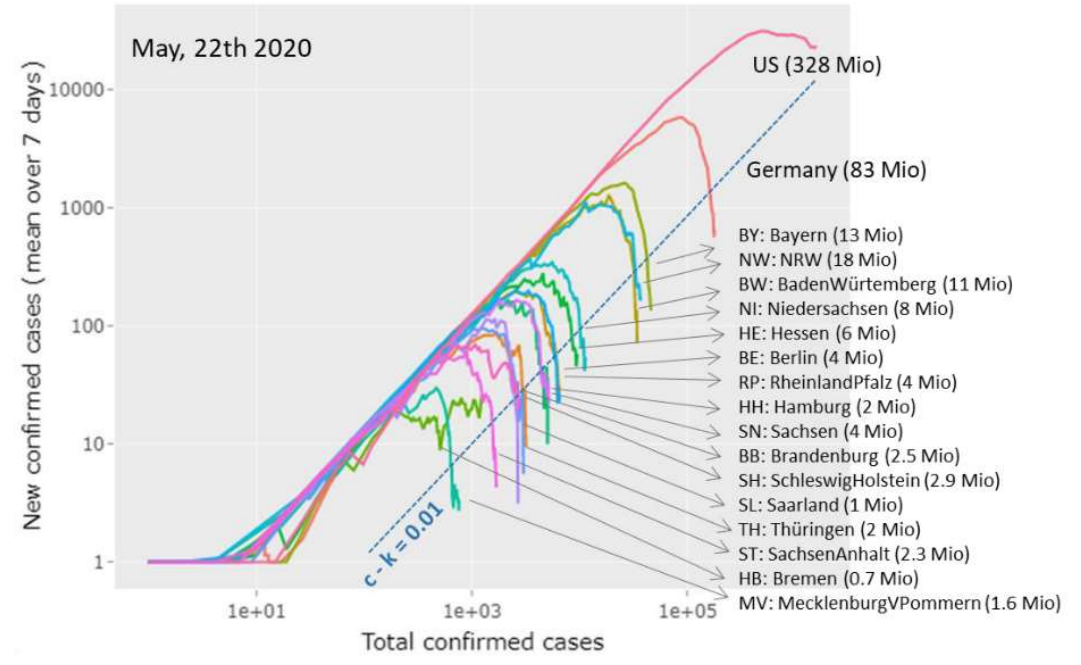

b) Infections per resident

c) Epicurves (cumulative numbers)
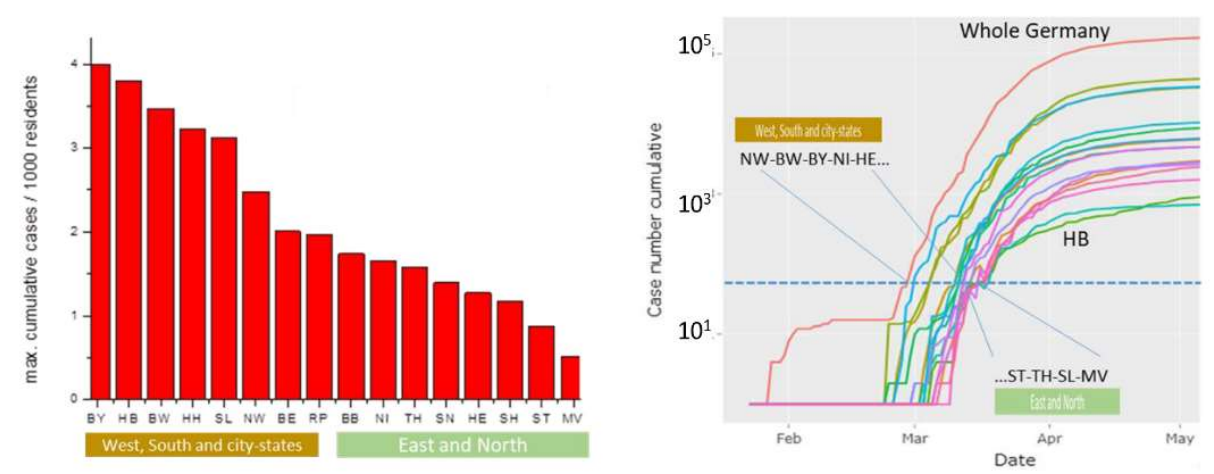

Figure 8. Covid-19 in Germany: (a) 'Rise-fall' trajectories across German states: The trajectories of German states resemble that of whole Germany indicating similar dynamics of Covid-19 across Germany. Trajectory of US is shown for comparison. (b) The relative maximum cumulative number of infected cases (per 1000 residents of the respective states divides clearly into states from West and South Germany and states in the East and North of Germany. City states (BE, HH, HB) are found in the former group. (c) Epi-curves (cumulative case numbers as a function of time) reveal that epidemic arrived earlier in Western and Southern states mostly by a few days compared with the Eastern and Northern ones. The curve of the city state Bremen (HB) slightly differs from that of the other ones. 

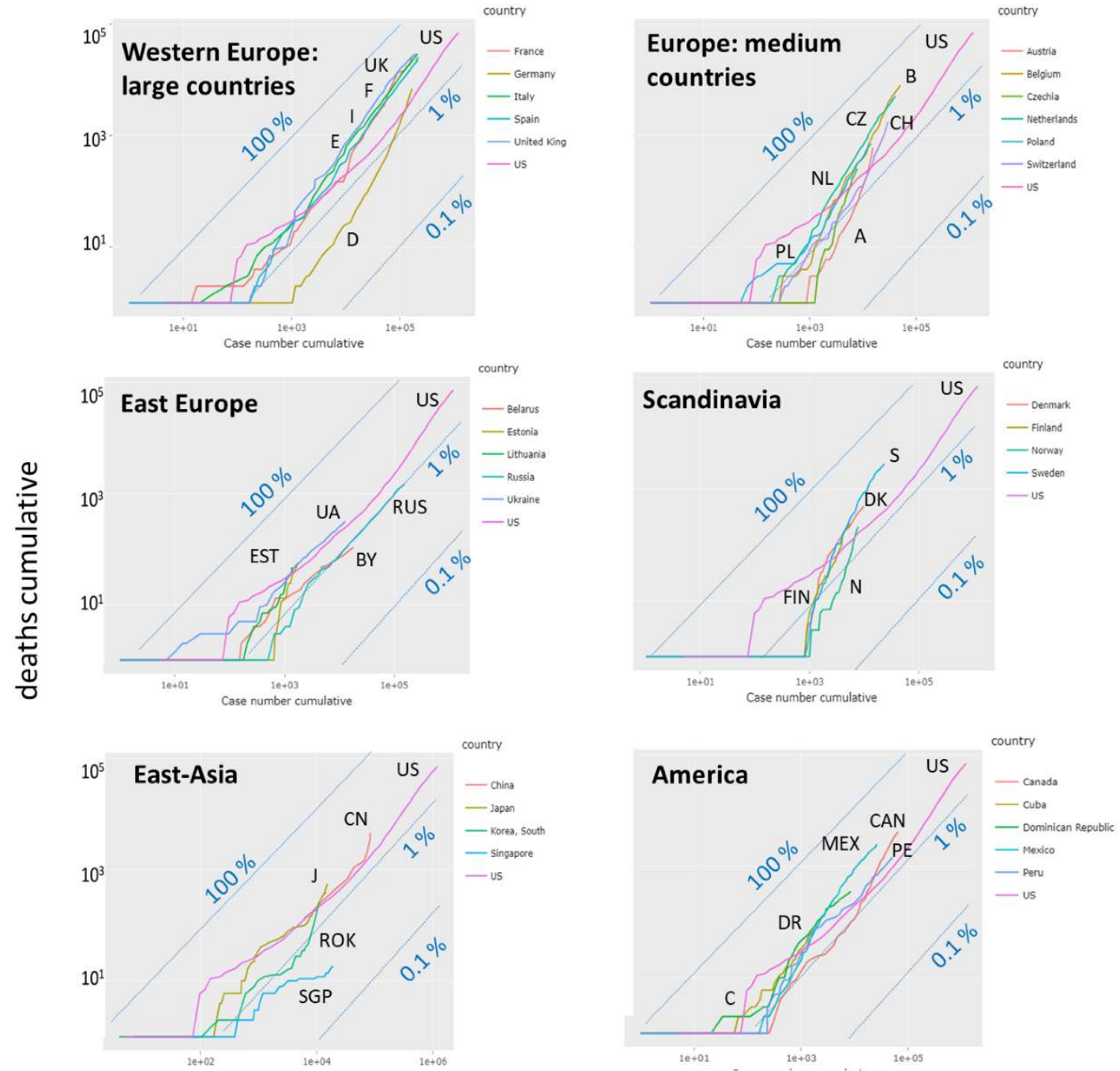

cases cumulative

Figure 9. Mortality trajectories show cumulative number of deaths versus cumulative number of infections. The diagonal line $(\mathrm{y}=\mathrm{x}$ ) refers to $100 \%$, parallel lines to $1 \%$ and $0.1 \%$ (number of deaths per 100 cases). The mortality trajectory of the US is shown in all plots for comparison.

\section{Discussion}

We here presented the 'Covid-19 trajectory viewer', which generates a series of trajectories and plots based on public available Covid19 data. It enables the comparison between epidemic development with country-wise resolution worldwide. Trajectories are based on two types of counts, namely the number of infected and of removed (recovered and died) individuals. Plots use either these counts directly, their cumulative values or increments per day and combine them in different ways, which allows to inspect the actual state of the epidemic under different perspectives.

In addition, the monitoring tool enables calculation and visualization of derived parameters, namely the effective transmission and recovery rate factors and the effective reproduction number. They estimate the transmission and removal 'power' as basic characteristics showing whether epidemic growths or declines. Changes of these parameters during epidemic development reflect different factors affecting the dynamics of epidemic, namely: (i) the possible consequences of NPIs, (ii) eventually growing immunity due to decaying numbers of susceptible individuals, and, (iii) also differences in the census methods of counting and reporting data between different countries. Our monitoring metric is sensitive for subtle alterations of the dynamics of the epidemic making it suitable to estimate the 
effectivity of NPIs and to serve as 'seismometer' for secondary outbreaks to early indicate such events (Figure 10).
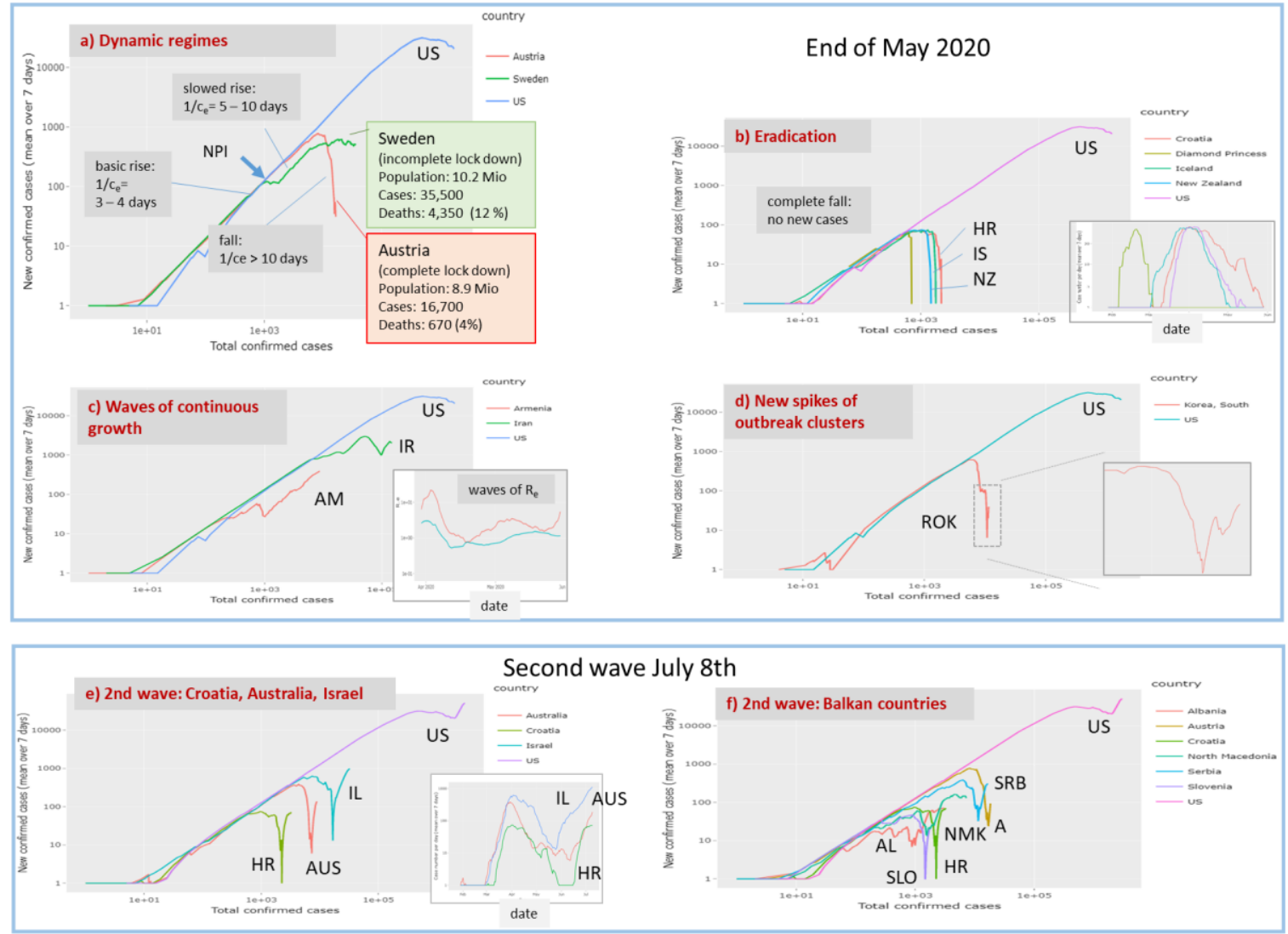

Figure 10. Example trajectories indicating different dynamic regimes of Covid-19: (a) Basic rise and falling regimes refer to transmission intervals of 3-4 and more than 10 days, respectively. They were observed in European countries under complete lock down such as Austria. Incomplete lock down as applied in Sweden only slowed down spread of epidemic. It associates with roughly two times more infections and a more than four-fold death-toll. (b) Eradication of epidemic can be expected in island states (Iceland, New Zealand) and other relatively small countries (e.g., Croatia) showing disappearance of new cases two to three months after the outbreak (see epi-curves in the insertion). Another example of eradication is Covid-19 spread at the Princess Diamond cruise liner with about 760 infections. (c) Wave-like up and downs of epidemic were observed in Armenia and Iran. The trajectories transform into wave-like oscillations of the effective reproduction number (insertion). (d) A new spike of cases is seen in the trajectory of South Korea. (e) The start of a 2nd wave becomes evident in the first week of July in selected countries, especially, (f) at the Balkan. The trajectory for US is shown for comparison showing also indications of a second wave.

Three possible future pandemic scenarios for Covid-19 dynamics have been suggested based on previos influenca courses [1], firstly, 'peaks and valleys' where the first big wave in spring 2020 is followed by repetitive smaller waves with geographic specifics depending on local NPIs. Secondly, the 'fall peak' suggesting a large secondary peak in the fall and winter of 2020; and, third, a 'slow burn' of ongoing transmission and case occurrence, but without a clear wave pattern, again with geographic variations affected by the degree of mitigation measures in place in various areas. The first scenario is always reality, but also the others will be possible. Trajectories and rate factor curves provide an instrument to distinguish the different scenarios. A recent forecasting model based on herd immunity end point predicts that effective NPIs are prone to induce a second wave of infections [39]. 
Thereby, one has to keep in mind that data refer to visible, symptomatic Covid19 cases. Un-symptomatic cases remain usually undetected and can exceed the number of symptomatic ones considerably. A recent publication shows that more than $80 \%$ of all positively tested Covid-19 cases on a cruise liner did not show any symptoms, raising questions about the true prevalence of "silent" infections [40] with possible consequences for the immunization dynamics in a population. Another analyses estimate that the true number of infections exceeds the reported ones up to a factor of ten [29] (and references cited therein). Moreover, our simple monitoring does not explicitly consider heterogeneities of the spread of the epidemic in a population (e.g., cities versus countryside, elderly versus younger, obese versus non-obese, men versus women, hospitalized versus non-hospitalized, symptomatic versus asymptomatic, highly exposed professions versus less exposed ones, etc.) [41]. Such effects are hidden in the data and can be considered in terms of the trajectory approach by using more detailed data, e.g., by stratifying populations geographically, with respect to professions, age, symptoms etc. and/or by applying more elaborated models.

Our visualization in terms of trajectories and derived rate factors and their interpretation is based on the simple SIR model dividing the visible population into three types of individuals. Such three-state models have been widely and successfully used in many areas of sciences to describe different kinds of dynamics, ranging from elementary reaction kinetics in chemistry to photo-physics, molecular transformations in biology and many other fields. The basic assumption behind the SIR model is the mass action law, claiming that changes of the population of a state directly relates to its population number. The different trajectories visualize this relationship by plotting changes of newly infected or removed individuals as functions of the number of cumulative or currently infected individuals. The double logarithmic scaling of the axes accounts for the fact that the solution of the ordinary differential equations behind the SIR model predicts exponential dynamics in important limiting regimes such as the early or late outbreak limits, which in turn, suggest linear courses of the trajectories. This way the obtained trajectories reflect a virtually common maximum transmission rate in the exponential growth phase in many countries suggesting that each infected individual infects another one every two-three days (Figure 10a). The initial growth is followed by down-steps and parallel shifted lines, which are indicative for exponential growth with reduced transmission rates (e.g., transfer of infection between two individuals every five days). Downturns of different sharpness indicate markedly reduced spread dynamics and eventually halt of the epidemic in terms of falling courses if transmission frequency reaches a level of more than only one per ten days.

The close temporal relatedness between slowing down of the transmission dynamics and the dates when measures of the NPI-type were taken suggests causal relations and shows that an associated 'falling' regime can be monitored using the trajectory approach. The NPI result in dropping transmission rates and reproduction numbers where the steepness of decay in Europe is larger for countries such as Austria and Germany, which were hit by the main infection wave a few weeks later than Italy, France and Spain showing slower decays. Early NIPs on a relative time scale with respect to growth dynamics obviously facilitate faster slowing down afterwards. So-called 'complete lockdown' measures seems to be an essential measure for stopping epidemic despite considerable differences between countries, e.g., in handling go-out restrictions ('Ausgangssperre', relatively moderate rules in Germany versus strong ones in Italy, Spain and France). The Swedish model seems to fail regarding transmission dynamics conceding further expanding epidemic and high death toll (Figure 10a). The recent lesson from the Spanish epidemic indicates that 'any proposed approach to achieve herd immunity through natural infection is not only highly unethical, but also unachievable' [42].

Our trajectories show that lowering a of transmission rates by more than $50-70 \%$ compared with its maximum, intrinsic value, is required to stop epidemic and to turn it into the decaying regime. A recent modelling study of COVID-19 clinical progression and transmission network structure on the outcomes of social distancing interventions shows that the strength of within-household transmission is a critical determinant of success, governing the timing and size of the epidemic peak, and also gives rise to a delay between the date when measures were taken and the decay of infections [43]. 
Joint plotting of trajectories using the Covid-19 viewer shows that at present majority of East Asian (China, South Korea, Singapore) and European countries are in the falling regime, while most American and South Asian countries are in the exponential growth phase. Epidemic seems virtually eradicated in the island states New Zealand, Iceland but also other small countries such as Croatia in a similar way as observed at 'Diamond Princess' Cruise Liner held under isolation (Figure 10b). It shows that isolation in combination with strong NPIs and extensive testing [29] effectively stop the epidemic. On the contrary, slowing down but still exponential growth are seen in other small and relatively isolated countries such as Armenia (surrounded by mountains and with closed borders to part of its neighbouring countries). Wavelike ups and downs as seen for Iran indicate repeated waves of a growing epidemic (Figure 10c). New outbreak clusters become evident as spiked upturns in the falling regime as indicated presently for South Korea (Figure 10d).

Parts e and $\mathrm{f}$ of Figure 10 show trajectories of countries (Israel, Balkan countries) showing a second wave about 3 weeks later (8 July), which reflects that a state near eradication (Croatia, Israel) can switch into a new outbreak within a few weeks if NPIs are locked up too fast and/or too widely. It shows that with a large majority of the population being infection naive, virus circulation can quickly return to early pandemic dimensions in a second wave once measures are lifted [42].

The removal rate factor is a second, important characteristic of Covid-19 dynamics, which additively composes of recovery and death rates, where the former number is dominating. Removal rates can differ by a factor of two-to-ten between different countries (e.g., Germany and Austria versus Spain and France, Figure S3) for unknown reasons. Possible explanations are specifics of the recovery process due to healthcare measures applied and/or epidemiological factors such as ageand/or health-risk of the respective populations. Also counting criteria of recovered individuals are another, possibly more relevant factor, which can differ between countries [29]. Often census agencies apply recovery counting algorithms (e.g., in Germany by assuming recovery two weeks after infection if no other information is available) presumably biasing estimation of removal rate factors. Moreover, also counting of deaths is census-dependent. On the other hand, the initially low but afterwards increasing mortality rates in Austria and Germany can be rationalized by the increasing age of infected individuals (disease was initially spread in communities of younger persons). Thus, comparing trajectories supports detection of differences of recovery and mortality rates between countries for subsequent analysis of the possible reasons.

\section{Conclusions}

The Covid-19 pandemic has developed in different phases around the world ranging from exponential growth to decaying regimes, partly until eradication, and, in some cases, starting second waves of epidemic from region to region and from country to country. It is characterized by high dynamics, which necessitate prompt monitoring to evaluate the outcome of NPI measures in either, 'lockdown' or 'lock up' direction to indicate improvement or worsening in terms of suited metrics such as increasing or decreasing numbers of cases, rate factors or reproduction numbers. The Covid-19 viewer provides this information in the worldwide context on a daily actualized basis. We understand our report as a worked example reflecting aspects of the pandemic mainly in May 2020, but also until acceptance in July, which supports future monitoring using the Covid-19 viewer as a sort of working instruction. For practical use we recommend: (i) Choosing 1-5 countries of interest and US and/or worldwide cases as reference(s) and inspecting their rise-fall trajectories. Figure 11 provides a simple evaluation scheme by dividing the 'phase space' into three sample-regions. The trajectories enable extracting the number of actually new cases and also to evaluate the course of epidemic as fast or slow growing, decaying or wavelike. (ii) If one is interested in more detailed numbers (currently infected, removed, deaths) then the respective axes in the 'custom trajectory' part for the same countries should be chosen and then the selection changed, if appropriate. One can compare, for example, fatality rates and their trends. (iii) If one is interested in rate constants for modelling tasks then transmission and removal rates can be inspected in the respective part. 
Many aspects of the Covid-19 pandemic are still not completely understood. This includes dark figures of infections, detailed spreading mechanisms and associated socio-economic, politic and health factors. Here more studies reasoning differences between regions and countries are required. The trajectory approach complements epi-curve reporting by bridging the gap to modelling methods. Inspection and comparison of the trajectories and of the time courses of rate factors extracted are expected to inspire development of substantiated hypotheses and elaboration of improved models to better understand mechanisms of epidemic spread and decay and theirs specific in different countries and regions. Overall, the viewer thus provides a snapshot of the pandemic in time and space, reflecting the geographical variability and the dynamics of Covid-19 under the circumstances in which it is done.

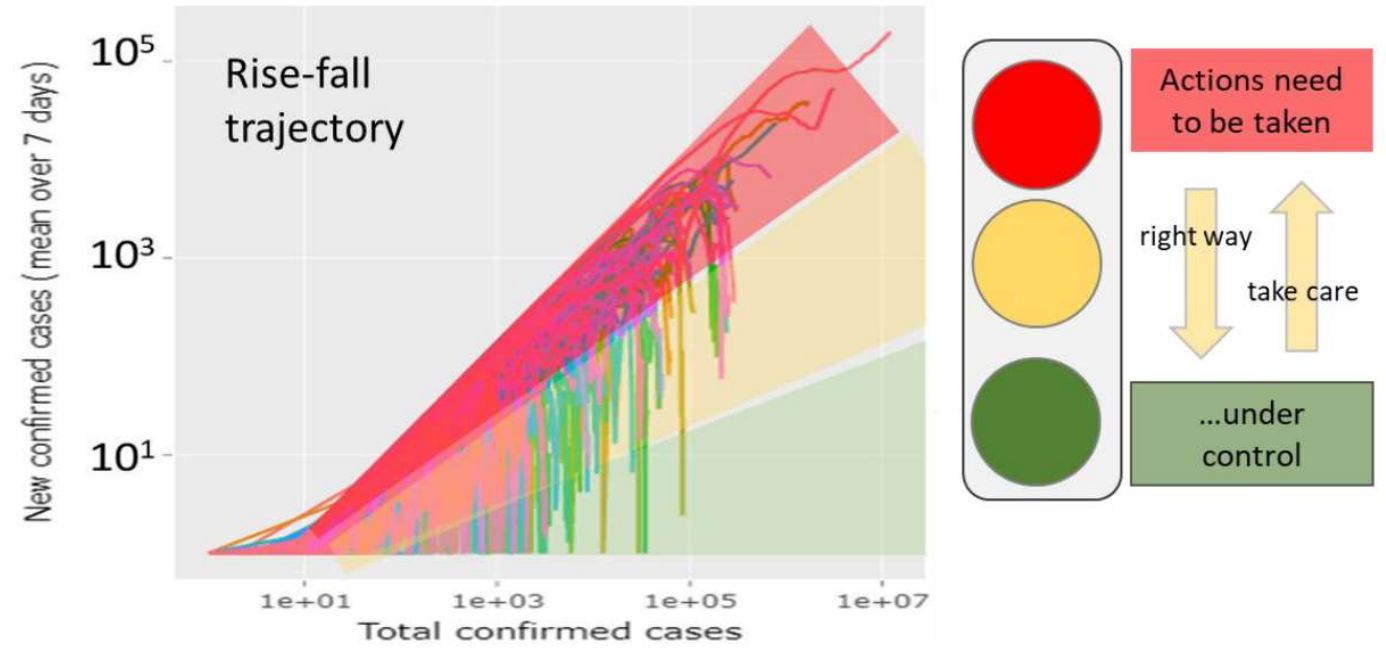

Figure 11. Traffic light dashboard for evaluating the state of the epidemic. The rise-fall trajectories provide the 'phase-space' of pandemic roughly dividing countries into ones that need to take actions, countries where epidemic is under control and countries that are at the right way if trajectory is falling or that have to take care if trajectory is rising (see also Figure S6).

Supplementary Materials: These are available online at http://www.mdpi.com/1999-4915/12/7/777/s1.

Author Contributions: Conceptualization and methodology, H.B. and H.L.-W.; software, H.L.-W.; formal analysis, M.S.; writing, H.B. All authors have read and agreed to the published version of the manuscript.

Funding: This research received no external funding. Publication was supported financially by the DFG-publication fund of University Library of the University of Leipzig.

Conflicts of Interest: The authors declare no conflict of interest.

\section{References}

1. Moore, K.A.; Lipsitch, M.; Barry, J.M.; Osterholm, M.T. COVID-19: The CIDRAP Viewpoint: Part 1: The Future of the COVID-19 Pandemic: Lessons Learned from Pandemic Influenza; Center for Infectious Disease Research and Policy (CIDRAP): Minneapolis, MN, USA, 2020. Available online: https://www.cidrap.umn.edu/sites/ default/files/public/downloads/cidrap-covid19-viewpoint-part1_0.pdf (accessed on 17 May 2020).

2. James, J.J. COVID-19: From Epidemic to Pandemic. Disaster Med. Public Health Prep. 2020. [CrossRef]

3. Hopkins, J. Coronavirus Ressource Centre. 2020. Available online: https://coronavirus.jhu.edu/ (accessed on 7 July 2020).

4. Roser, M.; Ritchie, H.; Ortiz-Ospina, E.; Hasell, J. Coronavirus Pandemic (COVID-19). 2020. Available online: https://ourworldindata.org/coronavirus (accessed on 17 May 2020).

5. Financial Times. Coronavirus Tracked: The Latest Figures as Countries Fight to Contain the Pandemic. 2020. Available online: https://www.ft.com/content/a26fbf7e-48f8-11ea-aeb3-955839e06441 (accessed on 17 May 2020).

6. The COVID Tracking Project. 2020. Available online: https://covidtracking.com/ (accessed on 17 May 2020). 
7. Smith, J.P. Comparison of COVID-19 case and death counts in the United States reported by four online trackers: January 22-May 31, 2020. medRxiv 2020. [CrossRef]

8. Gostic, K.M.; McGough, L.; Baskerville, E.; Abbott, S.; Joshi, K.; Tedijanto, C.; Kahn, R.; Niehus, R.; Hay, J.A.; De Salazar, P.M.; et al. Practical considerations for measuring the effective reproductive number, Rt. medRxiv 2020. [CrossRef]

9. Bock, W.; Adamik, B.; Bawiec, M.; Bezborodov, V.; Bodych, M.; Burgard, J.P.; Goetz, T.; Krueger, T.; Migalska, A.; Pabjan, B.; et al. Mitigation and herd immunity strategy for COVID-19 is likely to fail. medRxiv 2020. [CrossRef]

10. Barbarossa, M.V.; Fuhrmann, J.; Heidecke, J.; Vinod Varma, H.; Castelletti, N.; Meinke, J.H.; Krieg, S.; Lippert, T. A first study on the impact of current and future control measures on the spread of COVID-19 in Germany. medRxiv 2020. [CrossRef]

11. Donsimoni, J.R.; Glawion, R.; Plachter, B.; Waelde, K. Projecting the Spread of COVID19 for Germany. medRxiv 2020. [CrossRef]

12. Flaxman, S.; Mishra, S.; Gandy, A.; Unwin, H.J.T.; Coupland, H.; Mellan, T.A.; Zhu, H.; Berah, T.; Eaton, J.W.; Guzman, P.N.P.; et al. Estimating the number of infections and the impact of non-pharmaceutical interventions on COVID-19 in European countries: Technical description update. arXiv 2020, arXiv:2004.11342.

13. Khailaie, S.; Mitra, T.; Bandyopadhyay, A.; Schips, M.; Mascheroni, P.; Vanella, P.; Lange, B.; Binder, S.; Meyer-Hermann, M. Estimate of the development of the epidemic reproduction number Rt from Coronavirus SARS-CoV-2 case data and implications for political measures based on prognostics. medRxiv 2020. [CrossRef]

14. Dandekar, R.; Barbastathis, G. Quantifying the effect of quarantine control in Covid-19 infectious spread using machine learning. medRxiv 2020. [CrossRef]

15. Biswas, K.; Khaleque, A.; Sen, P. Covid-19 spread: Reproduction of data and prediction using a SIR model on Euclidean network. arXiv 2020, arXiv:2003.07063.

16. Engbert, R.; Rabe, M.M.; Kliegl, R.; Reich, S. Sequential data assimilation of the stochastic SEIR epidemic model for regional COVID-19 dynamics. medRxiv 2020. [CrossRef]

17. Maier, B.F.; Brockmann, D. Effective containment explains subexponential growth in recent confirmed COVID-19 cases in China. Science 2020, 368, 742-746. [CrossRef]

18. Soucy, J.-P.R.; Sturrock, S.L.; Berry, I.; Westwood, D.J.; Daneman, N.; MacFadden, D.R.; Brown, K.A. Estimating effects of physical distancing on the COVID-19 pandemic using an urban mobility index. medRxiv 2020. [CrossRef]

19. Chen, Y.-C.; Lu, P.-E.; Chang, C.-S.; Liu, T.-H. A Time-dependent SIR model for COVID-19 with Undetectable Infected Persons. arXiv 2020, arXiv:2003.00122.

20. Dehning, J.; Zierenberg, J.; Spitzner, F.P.; Wibral, M.; Neto, J.P.; Wilczek, M.; Priesemann, V. Inferring change points in the COVID-19 spreading reveals the effectiveness of interventions. arXiv 2020, arXiv:2004.01105.

21. Chernyshev, A. Autocatalytic Model for Covid-19 Progression in a Country. medRxiv 2020. [CrossRef]

22. Pell, B.; Kuang, Y.; Viboud, C.; Chowell, G. Using phenomenological models for forecasting the 2015 Ebola challenge. Epidemics 2018, 22, 62-70. [CrossRef]

23. Wangping, J.; Ke, H.; Yang, S.; Wenzhe, C.; Shengshu, W.; Shanshan, Y.; Jianwei, W.; Fuyin, K.; Penggang, T.; Jing, L.; et al. Extended SIR Prediction of the Epidemics Trend of COVID-19 in Italy and Compared with Hunan, China. Front. Med. 2020, 7, 169. [CrossRef]

24. Su, L.; Hong, N.; Zhou, X.; He, J.; Ma, Y.; Jiang, H.; Han, L.; Chang, F.; Shan, G.; Zhu, W.; et al. Evaluation of the Secondary Transmission Pattern and Epidemic Prediction of COVID-19 in the Four Metropolitan Areas of China. Front. Med. 2020, 7, 171. [CrossRef]

25. Taghizadeh, L.; Karimi, A.; Heitzinger, C. Uncertainty Quantification in Epidemiological Models for COVID-19 Pandemic. medRxiv 2020. [CrossRef]

26. Timothy, J.J.; Holla, V.; Meschke, G. Multiscale dynamics of COVID-19 and model-based recommendations for 105 countries. medRxiv 2020. [CrossRef]

27. Kevorkian, A.; Grenet, T.; Gallee, H. Tracking the Covid-19 pandemic: Simple visualization of the epidemic states and trajectories of select European countries \& assessing the effects of delays in official response. medRxiv 2020. [CrossRef]

28. Chang, W.; Cheng, J.; Allaire, J.; Xie, Y.; McPherson, J. Shiny: Web Application Framework for R; R Package Version; 2017. Available online: http://shiny.rstudio.com/tutorial/ (accessed on 15 April 2020). 
29. Kuster, A.C.; Overgaard, H.J. A novel comprehensive metric to assess COVID-19 testing outcomes: Effects of geography, government, and policy response. medRxiv 2020. [CrossRef]

30. Fonfria, E.S.; Vigo, M.I.; Garcia-Garcia, D.; Herrador, Z.; Navarro, M.; Bordehore, C. Essential epidemiological parameters of COVID-19 for clinical and mathematical modeling purposes: A rapid review and meta-analysis. medRxiv 2020. [CrossRef]

31. Flaxman, S.; Mishra, S.; Gandy, A.; Unwin, H.J.T.; Mellan, T.A.; Coupland, H.; Whittaker, C.; Zhu, H.; Berah, T.; Eaton, J.W.; et al. Estimating the effects of non-pharmaceutical interventions on COVID-19 in Europe. Nature 2020. [CrossRef]

32. Dehning, J.; Zierenberg, J.; Spitzner, F.P.; Wibral, M.; Neto, J.P.; Wilczek, M.; Priesemann, V. Inferring change points in the spread of COVID-19 reveals the effectiveness of interventions. Science 2020, 369, eabb9789. [CrossRef]

33. Brauner, J.M.; Mindermann, S.; Sharma, M.; Stephenson, A.B.; Gavenčiak, T.; Johnston, D.; Salvatier, J.; Leech, G.; Besiroglu, T.; Altman, G.; et al. The effectiveness and perceived burden of nonpharmaceutical interventions against COVID-19 transmission: A modelling study with 41 countries. medRxiv 2020. [CrossRef]

34. Soltesz, K.; Gustafsson, F.; Timpka, T.; Jaldén, J.; Jidling, C.; Heimerson, A.; Schön, T.; Spreco, A.; Ekberg, J.; Dahlström, Ö.; et al. Sensitivity analysis of the effects of non-pharmaceutical interventions on COVID-19 in Europe. medRxiv 2020. [CrossRef]

35. Bar-On, Y.M.; Flamholz, A.; Phillips, R.; Milo, R. Science Forum: SARS-CoV-2 (COVID-19) by the numbers. eLife 2020, 9, e57309. [CrossRef] [PubMed]

36. Casanova, J.-L.; Su, H.C. A global effort to define the human genetics of protective immunity to SARS-CoV-2 infection. Cell 2020, 181, 1194-1199. [CrossRef]

37. Petrilli, C.M.; Jones, S.A.; Yang, J.; Rajagopalan, H.; O’Donnell, L.F.; Chernyak, Y.; Tobin, K.; Cerfolio, R.J.; Francois, F.; Horwitz, L.I. Factors associated with hospitalization and critical illness among 4103 patients with COVID-19 disease in New York City. medRxiv 2020. [CrossRef]

38. Docherty, A.B.; Harrison, E.M.; Green, C.A.; Hardwick, H.E.; Pius, R.; Norman, L.; Holden, K.A.; Read, J.M.; Dondelinger, F.; Carson, G.; et al. Features of 16,749 hospitalised UK patients with COVID-19 using the ISARIC WHO Clinical Characterisation Protocol. medRxiv 2020. [CrossRef]

39. Ackland, G.J.; Rice, K.; Wynne, B.M.; Martin, V. The long term predictions from Imperial College CovidSim Report 9. medRxiv 2020. [CrossRef]

40. Ing, A.J.; Cocks, C.; Green, J.P. COVID-19: In the footsteps of Ernest Shackleton. Thorax 2020. [CrossRef]

41. Takagi, H.; Kuno, T.; Yokoyama, Y.; Ueyama, H.; Matsushiro, T.; Hari, Y.; Ando, T. Risk and protective factors of SARS-CoV-2 infection-Meta-regression of data from worldwide nations. medRxiv 2020. [CrossRef]

42. Eckerle, I.; Meyer, B. SARS-CoV-2 seroprevalence in COVID-19 hotspots. Lancet 2020. [CrossRef]

43. Nande, A.; Adlam, B.; Sheen, J.; Levy, M.Z.; Hill, A.L. Dynamics of COVID-19 under social distancing measures are driven by transmission network structure. medRxiv 2020. [CrossRef] 